\title{
Desenvolvimento e implementação de uma metodologia para troca rápida de ferramentas em ambientes de manufatura contratada
}

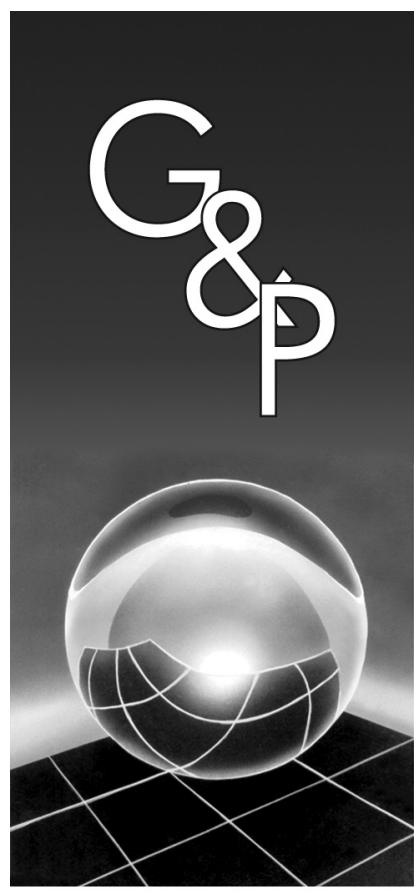

\author{
Samuel Vieira Conceição \\ lana Araújo Rodrigues \\ Andressa Amaral Azevedo \\ João Flávio Almeida \\ Fabrício Ferreira \\ Adriano Morais
}

Resumo

Este artigo apresenta o desenvolvimento de uma metodologia de redução do tempo de preparação de máquina (tempo de changeover) para ambientes voláteis de manufatura contratada do setor eletroeletrônico e de informática.. As empresas desse setor fabricam uma grande variedade de produtos caracterizados por um alto valor agregado, curto ciclo de vida e instabilidade da demanda, exigindo flexibilidade das empresas para acompanhar o dinamismo do mercado. Com isso, a produção enxuta surge como uma alternativa, já que tem como características fluxo contínuo de produção, altos níveis de qualidade, redução de custos e agilidade na introdução de mudanças, fatores primordiais para se atingir a flexibilidade. O presente artigo tem como foco a redução do tempo de troca de ferramentas em uma linha de produção que utiliza a tecnologia de montagem em superfície conhecida como SMT (Surface Mount Technology). A diminuição desse tempo é relevante, pois possibilita a redução da necessidade de estoques intermediários; aumento da flexibilidade de produção com lotes e lead times menores; aumento de produtividade; respostas rápidas a mudanças de volume; e velocidade na programação da produção. A metodologia utilizada foi a pesquisa ação. O artigo traz contribuições teóricas e práticas além da aplicação da metodologia desenvolvida no setor de manufatura contratada. O método desenvolvido possibilitou ganhos importantes de flexibilidade de resposta a alterações de variedade e volume, traduzidos em ganhos de flexibilidade, produtividade e financeiros.

Palavras-chave: Redução de changeover. Produção enxuta. Manufatura contratada.

\section{Introdução}

Nos ambientes caracterizados por alta variedade de produtos e baixo volume de produção, torna-se imprescindível o desenvolvimento de competências distintivas relacionadas à flexibilidade de mix e volume. Segundo Upton (1995), a flexibilidade pode ser definida de duas formas:

i. habilidade de trocar rapidamente a produção mobilidade que possibilita maior capacidade de resposta às variações da demanda -; e

ii. habilidade de produzir uma grande variedade de produtos. Boyle e Scherrer-Ratje (2009) evidenciam que as melhores práticas de manufatura enxuta são caracterizadas por: incorporar o aspecto flexibilidade na estratégia de manufatura da empresa; identificar as fontes potenciais de incerteza para a manufatura; e identificar a capacidade da manufatura em identificar e reduzir as fontes de incertezas. A flexibilidade tem um papel importante na manufatura contratada, na qual os fabricantes de produtos eletrônicos estão inseridos em um ambiente altamente dinâmico e complexo, com demandas diversificadas e mercado evolucionário. Esse dinamismo se deve ao curto ciclo de vida dos produtos com altas taxas de inovações tecnológicas.

Uma característica relevante do setor eletroeletrônico é a predominância de um modelo organizacional em que as empresas detentoras da marca, denominadas OEM (Original Equipment Manufacturer), terceirizam a produção para as empresas que prestam serviços de manufatura, denominadas EMS (Electronic Manufacturing Services). 
As empresas de serviço eletrônico de manufatura EMS (Electronic Manufacturing Service) ou CEMS (Contract Electronic Manufacturing Services) são um caso especial de manufatura contratada (Contract Manufacturer - CM). Essas empresas tiveram suas origens nos anos 70, quando as grandes empresas eletrônicas americanas se viram ameaçadas pela competição dos países asiáticos. Em face à dificuldade de responder adequadamente a essa concorrência, optaram por terceirizar as atividades de manufatura dos produtos (STURGEON, 1997). Outro fator que levou à adoção da estratégia de manufatura contratada foi a grande velocidade na introdução de novos produtos. Os saltos ou rupturas tecnológicas advindas do surgimento de novos materiais, principalmente com a miniaturização dos componentes eletrônicos e as economias de escala possibilitaram reduções significativas nos custos de produção (e no preço final ao consumidor), aumentando a demanda pelos bens eletro, eletrônicos e de informática. Recentemente, as empresas japonesas também têm mostrado interesse na adoção dessa estratégia para fabricação de automóveis (WEBER, 2002). Porém, essa opção não é frequente em OEMs (Original Equipment Manufacturer) que possuem características de produção em grandes volumes e pequena variedade de produtos (WEBER, 2002).

A função das empresas de manufatura contratada, inicialmente, era apenas fabricar produtos para as OEMs. Hoje, a presença de mercados mundiais ampliou esse escopo, tornando-as responsáveis, também, pela compra dos materiais, seleção de fornecedores e gerenciamento da cadeia de suprimentos (CHAN; CHUNG, 2002). Alguns dos elementos dessa mudança foram a redução do ciclo de vida dos produtos, o surgimento da manufatura enxuta e a pressão por respostas rápidas às demandas dos consumidores. $\mathrm{O}$ foco das OEMs está nas funções que apresentam relação direta com a manutenção do mercado, isto é, o desenvolvimento de produtos e marketing, principalmente no que se refere à notoriedade da marca (STURGEON, 1997; HADAYA; LEFEBVRE; LÉGER, 2000; TANEL, 2005).

Desde o início deste movimento de contratação de manufatura, as EMSs obtiveram enorme crescimento. Em 2008, as 50 maiores empresas foram responsáveis por uma venda de U\$158,5 bilhões, o que representa um crescimento considerável em comparação ao faturamento de U\$ 109,2 bilhões de 2005 (MMI, 2009). A divisão das atividades entre as empresas detentoras da marca (OEM) e as de manufatura contratada (CM) é vantajosa para ambas as partes. As OEM possuem vantagens quanto à rapidez na introdução de novos produtos, enquanto que as EMS, ao se especializarem nesse tipo de serviço, conseguem custos de produção mais baixos que as OEMS. Além disso, seus clientes geralmente são concorrentes diretos, o que proporciona um aprendizado na fabricação dos produtos, possibilitando o aumento do nível de serviço oferecido aos consumidores finais das OEMs por meio da manufatura (HADAYA; LEFEBVRE; LÉGER, 2000). Esse nível de serviço pode ser medido por indicadores como custos, flexibilidade, produtividade, velocidade, e qualidade dos produtos manufaturados. As empresas de manufatura contratadas de sucesso serão aquelas que conseguirem ser enxutas em todos os aspectos, sendo hábeis na coordenação dos diferentes serviços oferecidos aos clientes (TANEL, 2005). Dessa forma, iniciativas na adoção de técnicas da produção enxuta têm sido mais evidenciadas no setor de manufatura contratada (FARLOW, 2005). Diante desse contexto, a produção enxuta é uma metodologia que pode conferir à empresa características de flexibilidade, uma vez que possibilita uma produção estável, com custos reduzidos, baixos níveis de estoques, qualidade assegurada e agilidade na incorporação de mudanças no sistema produtivo. Um dos grandes desafios relacionados à flexibilidade tem sido a união da flexibilidade aliada a padrões mínimos de produtividade e, nesse aspecto, o SMED traz uma contribuição significativa.

O objetivo deste artigo é desenvolver uma metodologia de implementação do SMED (Single Minute Exchange of Die) - Troca Rápida de Ferramenta em ambientes de manufatura contratada -, incorporando uma discussão teórica sobre o tema, com ênfase no ambiente de produção. $\mathrm{O}$ artigo também ilustra a aplicação da metodologia desenvolvida em uma empresa de manufatura contratada. O artigo está estruturado da seguinte forma: a seção 2 discute o estado da arte dos principais assuntos abordados; a seção 3 destaca a metodologia de pesquisa adotada para o desenvolvimento do trabalho; a seção 4 contextualiza a empresa estudada e descreve a metodologia SMED desenvolvida, bem como, os resultados alcançados com sua implementação; a seção 5 apresenta as implicações teóricas e práticas deste estudo; e, por último, a seção 6 mostra as conclusões e considerações finais do trabalho desenvolvido.

A contribuição científica está no desenvolvimento de uma metodologia de redução do tempo de changeover para ambientes complexos e dinâmicos de manufatura contratada caracterizados pela alta volatilidade da demanda, baixo volume de produção, alta variedade de produtos e margens de lucro apertadas. A metodologia desenvolvida pode ser aplicada a um número significativo de empresas que atuam nesse setor, principalmente no Brasil, onde existe um esforço considerável do poder público para desenvolver o setor de produtos eletrônicos e de informática. $\mathrm{O}$ artigo discute aspectos relevantes do problema de flexibilidade na manufatura contratada, apontando as principais questões e soluções discutidas na literatura. A implementação se dá no nível técnico, isto é, no desenvolvimento de dispositivos e ferramentas em sistemas de produção de placas de circuito impresso com o objetivo de reduzir o tempo de changeover. Essa é outra contribuição importante no artigo, visto que na engenharia de produção, o problema tem sido discutido mais sob a ótica organizacional do que na proposição de soluções tecnológicas, como a abordada no presente artigo. 


\section{Revisão de literatura}

O sistema de produção enxuta tem sido intensivamente discutido na literatura, sobretudo por autores tais como Monden, 1994; Schonberger, 1982; Shingo, 1989; Hall, 1983. Segundo Bayou e Corvin (2008), apesar das inúmeras pesquisas relatadas na literatura nas últimas décadas, esse tema não está ainda completamente desenvolvido por duas razões: em primeiro lugar, falta uma definição geral que seja amplamente aceita pelos pesquisadores; em segundo, até o momento não existem publicações que tenham desenvolvido uma medida relativa e sistemática sobre sistema de produção enxuta. Shah e Ward (2007) também discutem o que consideram a inconsistência e confusão semântica com o termo produção enxuta. Numa revisão de literatura por meio de uma perspectiva histórica evolucionária, são discutidos os conceitos dados por diversos autores sobre o tema e técnicas de suporte a esse sistema de produção. Outra contribuição importante relativa à evolução e genealogia da produção enxuta é discutida em detalhes por Holweg (2007). Baseando-se em entrevistas com os principais autores e empresas sobre produção enxuta e em pesquisa bibliográfica, o autor apresenta um histórico sobre a genealogia e disseminação do que considera um dos principais paradigmas dos últimos tempos: o sistema de produção enxuta.

A produção enxuta é caracterizada por um curto lead time, alto nível de qualidade e eficiente uso dos recursos, com a participação de toda a empresa, desde a alta direção até os operadores de chão de fábrica, que passam a ter maior autonomia, e colaboração dos fornecedores, principalmente, no desenvolvimento de produtos (WOMACK; JONES; DANIEL, 1992). Shingo (1989) a define como "um sistema de absoluta eliminação de desperdícios”, destacando alguns princípios como:

i Redução de custos pela eliminação de desperdícios qualquer atividade que não agrega valor ao produto final;

ii Just in Time (JIT) - suprimento com os itens corretos, nas quantidades exatas e no tempo certo;

iii Produção puxada - liberação da produção somente com a confirmação do pedido do cliente;

iv Redução dos tempos de setup/changeover - tempos curtos na preparação da linha quando da troca de produção;

v Eliminação de quebras e defeitos - prevenção de problemas em todo o processo;

vi Nivelamento da produção - balanceamento entre o volume de trabalho a ser executado com a capacidade (máquina/mão de obra) disponível; e

Automação. Åhlström (1998) enfatiza, ainda, os seguintes princípios da produção enxuta:

i Equipes multifuncionais com trabalhadores multifuncionais; ii Autonomia aos trabalhadores;

iii Líderes de equipe com função de conselheiros, motivadores e suporte;

iv Sistemas de informação vertical simples e confiável; e

v Melhoria contínua.

Pham et al. (2008) propõem um novo modelo que integra os conceitos de produção enxuta e produção ágil (agile manufacturing) com o marketing e as estratégias de inovação em produção, capaz de prover um novo sistema de gerenciamento da produção economicamente sustentável e capaz de operar de maneira eficiente em ambientes de mercados globalizados altamente competitivos. Pesquisa realizada por Boyle e Scherrer-Ratje (2009) evidenciam que as melhores práticas de manufatura enxuta são: incorporar o aspecto flexibilidade na estratégia de manufatura da empresa; identificar as fontes potenciais de incerteza para a manufatura; identificar a capacidade da manufatura em identificar e reduzir as fontes de incertezas. Outra pesquisa realizada com 27 empresas de manufatura enxuta, objetivando estudar as principais práticas realizadas nas áreas de processo e equipamento, planejamento e controle da produção, recursos humanos, projeto do produto, relações com fornecedores e relação com consumidores, constatou que a maioria das ações é tomada dentro do próprio ambiente da organização e que a efetivação de relações externas com fornecedores e consumidores é ainda um desafio a ser vencido (PANIZZOLO, 1998).

Krafcik (1988) argumenta que o desempenho da produção está também diretamente relacionado à política de gerenciamento adotada. Menciona, também, que a manufatura enxuta é altamente relevante para se conseguir, simultaneamente, altos níveis de produtividade, qualidade e flexibilidade dos produtos, embora sua implementação seja de risco, mas com altas chances de retorno do investimento. Os riscos são atribuídos ao fluxo contínuo de produção com baixos níveis de estoques, e a ocorrência de qualquer imprevisto acarreta a interrupção da linha de produção. Porém esses riscos podem ser amenizados com força de trabalho bem treinada e multifuncional, projeto de produtos com boas características de manufaturabilidade (fáceis de serem produzidos), com alta qualidade e bom suporte dos fornecedores (confiabilidade dos produtos e velocidade de entrega).

Pesquisas empíricas realizadas por Herron e Hicks (2008) evidenciam que o apoio da alta diretoria das empresas e que a habilidade dos facilitadores são fatores determinantes para o sucesso da implementação. Uma das condições necessárias para obter esse elevado desempenho no setor de manufatura contratada é a troca rápida de ferramentas com o objetivo de reduzir o tempo de changeover ou tempo de ajuste ou preparação das máquinas.

Segundo McIntosh et al. (1996), não há na literatura uma nomenclatura padronizada para definir tanto a palavra como 
os procedimentos de changeover. McIntosh et al. (1996) definem changeover como a soma do tempo de setup (tempo de parada da linha de produção para a troca de produto) e o tempo de run-up (tempo gasto para elevar e estabilizar a produção referente às taxas de produtividade e qualidade). Essa definição pode ser ainda complementada pela adição do tempo de run down (tempo gasto para reduzir a taxa de produção até o limite da parada completa da linha). Esse problema de terminologia e conceito também é discutido por Mileham et al., 1999, segundo os quais os processos de melhoria no tempo de changeover, geralmente, se dedicam apenas aos aspectos de setup desse, o que faz com que, muitas vezes, seja definido pelos autores como redução de setup (MILEHAM et al., 1999).

Os principais fatores motivadores da redução do changeover são:

1. Flexibilidade e redução de estoques - a redução do tempo de setup permite a produção de pequenos lotes e, consequentemente, aumento da variedade de produtos ofertados em menores quantidades;

2. Capacidade do gargalo - a redução do tempo de setup significa aumento da capacidade produtiva e;

3. Minimização de custos - uma porção do custo de um produto é determinada pelo custo de produção diretamente relacionado ao desempenho das máquinas, que terá menos tempo ocioso com a redução do tempo de setup (VAN GOUBERGEN; VAN LANDEGHEM, 2002).

As vantagens alcançadas com a redução do tempo de setup/changeover são: produção de pequenos lotes; redução do lead time; redução de estoques; aumento da qualidade; redução de desperdício e retrabalho; aumento da flexibilidade e responsividade; aumento de produtividade; conscientização das causas que geram erros e espera; e, também, aumento da disponibilidade dos equipamentos (DIABY, 2000; OHNO, 1997; MCINTOSH et al., 1996; MILEHAM et al., 1999; SHINGO, 1988, 1989).

Para Van Goubergen (2000), um setup de qualidade se sustenta em organização, método e aspectos técnicos dos equipamentos e ferramentas, sendo a motivação das pessoas envolvidas sua principal base. O setup ou changeover sofre influências de várias áreas da empresa e não somente da produção (VAN GOUBERGEN, 2000). Segundo Mileham et al. (1999), existem duas diferentes estratégias para redução de changeover: melhorar o sistema já existente ou criar um sistema totalmente diferente. A Tabela 1 apresenta vários aspectos importantes no processo de redução do tempo de changeover. Mileham et al. (1999), Van Goubergen e Van Landeghem (2002) e McIntosh et al. (1996), objetivando reduzir o excesso de esforço físico, ajustes e variedades no processo de changeover, fornecem regras a serem seguidas no desenvolvimento de novos equipamentos ou melhoria dos existentes. Para Van Goubergen e Van Landeghem (2002), o fabricante do equipamento deveria considerar, ainda na fase do projeto, aspectos de responsabilidade técnica, métodos e organização, possibilitando um setup padronizado e eficiente. No desenvolvimento de metodologia para a execução das atividades de changeover, o SMED (Single Minute Exchange of Die) é uma ferramenta bastante difundida. O SMED foi criado no Japão por Shigeo Shingo no começo dos anos 50, a partir da necessidade da diversificação de produtos em baixos volumes, o que aumenta o número de equipamentos envolvidos nos setups (MOXHAM; GREATBANKS, 2001). A maioria dos autores aborda a metodologia de Shingo ao se tratar da redução de setup/changeover, tais como: Sharma (2001), Agustin e Santiago (1996), Severson (1988), Moxham e Greatbanks (2001).

A redução de setup com o uso do SMED contém as seguintes etapas (Shingo, 1988, 1989):

i não fazer distinção entre setup interno (executado com a máquina parada) e externo (executado com a máquina em funcionamento);

ii separação do setup interno e externo;

iii conversão, ao máximo, do setup interno em externo;

vi aprimoramento do setup interno e externo. Sugere-se, ainda, para a redução do tempo de setup: uso de gabaritos intermediários para facilitar a preparação da máquina na troca de produção, realização de operações paralelas a fim de provocar economia de movimentos, padronização das funções das partes necessárias na operação de setup, uso de dispositivos de apertos funcionais, eliminação de ajustes e mecanização.

Monden (1994), além de apresentar um conjunto de técnicas para se alcançar a redução do setup com o uso do SMED, sugere algumas atividades a serem realizadas durante a implementação: conhecer as reais condições das atividades de setup, filmar para conhecer melhor as restrições de tempo e movimentos e documentar a rotina das operações padrão.

Apesar do tempo de setup estar frequentemente relacionado apenas à área de produção, ele também é reflexo das ações de outras áreas da empresa. Ao não fornecer infraestrutura apropriada e não treinar e realizar programas de conscientização da importância do curto tempo de setup, a gerência influencia diretamente no sucesso ou fracasso das iniciativas de redução do tempo de changeover (VAN GOUBERGEN, 2000). A área de desenvolvimento de equipamentos, ao desconsiderar a facilitação do processo de setup aos usuários, ou a área de compras na aquisição de equipamentos baseada somente nos custos de investimentos ou, ainda, na aquisição de um mesmo material com especificações diversas, também refletem no tempo de setup da empresa. A área de projeto do produto, durante a concepção do produto, nem sempre se preocupa com a eliminação de atividades de setup. Ao 
gerar ajustes demasiados para atender a suas especificações rigorosas, o setor de qualidade aumenta o tempo de setup, assim como faz a gerência de materiais ao não disponibilizar os materiais a tempo de uso ou ao fornecer lista de materiais inadequada. O sistema de manutenção ineficiente ou o planejamento e controle da produção mal elaborados, também refletem negativamente no tempo de setup. De forma análoga, o sistema de manutenção ineficiente também traz consequências negativas no tempo de setup e no planejamento e controle da produção.

A redução de changeover é o pré-requisito para possibilitar a produção em pequenos lotes. Se fosse possível a redução do tempo de changeover a zero, isso possibilitaria à empresa uma flexibilidade dinâmica e seria uma condição necessária para que ela fosse capaz de fabricar qualquer variedade de produtos.

\section{Metodologia}

A metodologia de pesquisa adotada na condução deste trabalho foi a pesquisa ação. Segundo Coughlan, P. e Coughlan, D. (2002), o termo pesquisa ação designa um tipo de pesquisa com base empírica que é concebida e realizada em estreita colaboração com uma ação contemplando muitas formas de pesquisa orientada à ação, indicando a grande diversidade em teoria e prática entre os pesquisadores que utilizam tal metodologia .

As dez características principais da pesquisa ação são (COUGHLAN, P.; COGHLAN, D. 2002): o pesquisador toma ação e não é mero observador; envolve dois objetivos principais: solucionar um problema e contribuir para a ciência; é interativa, isto é, ocorre cooperação e interatividade entre os envolvidos; objetiva desenvolver um entendimento sistêmico ou holístico; é fundamentalmente relacionada ao processo de mudança; requer um entendimento da estrutura étnica englobando valores e normas; pode incluir vários tipos de métodos de coleta de dados (técnicas quantitativas e qualitativas); requer uma ampla compreensão e entendimento do ambiente organizacional, condições, estrutura e dinâmica das operações; deve ser conduzida em tempo real, requer critérios próprios de qualidade para sua avaliação.

Várias abordagens são discutidas na literatura sobre pesquisa ação. Uma das metodologias mais bem conceitualmente construídas em pesquisa ação é o SSM (soft system methodology) ou metodologia de sistemas flexíveis ou metodologia de sistemas soft. Segundo Checkland (1981) e Checkland e Scholes (1990), o SSM é uma metodologia baseada no processo sistêmico cuja utilização enquanto instrumento de pesquisa pode ser utilizada para promover mudanças organizacionais em situações reais em que haja a ocorrência de ambientes ou situações consideradas complexas ou problemáticas. Apesar de inúmeros relatos de sucesso, a literatura também discute várias experiências mal sucedidas com o SSM. Nesse sentido, vários refinamentos nessa metodologia são propostos por Couprie et al. (1997) e Salner (1999).

Heale (2003) considera cinco diferentes etapas na aplicação da pesquisa ação: detecção do problema; planejamento das ações; execução das atividades; análise dos resultados; e reflexão e reformulação da teoria. Cada etapa é um ciclo no qual ocorre a ação direta seguida da reflexão crítica, avaliação e síntese antes da inclusão de mais alterações.

A Figura 1 descreve os principais passos da metodologia desenvolvida para a redução do tempo de changeover.

A implementação da metodologia desenvolvida foi conduzida com treinamentos teóricos e práticos no chão de fábrica. Inicialmente, foram treinados os integrantes da equipe "piloto" e a metodologia implementada na linha de produção teste. Posteriormente, os demais operadores do turno da equipe piloto, passando para os demais turnos e linhas de produção de forma sequencial.

A etapa de monitoramento foi conduzida por meio de observações, entrevistas com os trabalhadores e reuniões semanais. Esta etapa foi importante, uma vez que possibilitou readaptar o processo definido, eliminando falhas e incoerências e, também, levantar problemas que afetavam a atividade de changeover.

Essa etapa deve ser constante para acompanhar se o processo está sendo executado como proposto e, também, captar possibilidades de melhoria contínuas. O detalhamento dos passos desenvolvidos está ilustrado na Figura 2.

A metodologia acima foi desenvolvida para os ambientes de produção de placas de circuito impresso e montagem de produtos finais (impressoras e leitores de cartão de crédito).

\section{O Desenvolvimento e Implementação da Metodologia - SMED}

O SMED abaixo detalhada se refere à aplicação da metodologia adaptada de Shingo (1989). Essa adaptação se refere aos procedimentos particulares de desenvolvimento do

Tabela 1. Comparação das estratégias de redução de changeover.

\begin{tabular}{lcc}
\hline \multicolumn{1}{c}{ Aspectos } & $\begin{array}{c}\text { Apenas metodologia } \\
\text { (melhoria das } \\
\text { práticas existentes) }\end{array}$ & $\begin{array}{c}\text { Projeto e } \\
\text { metodologia } \\
\text { (novas práticas) }\end{array}$ \\
\hline $\begin{array}{l}\text { Tempo de alcance de } \\
\text { desempenho }\end{array}$ & Rápido & Longo \\
Esforço requerido & Baixo-médio & Médio-alto \\
Custo & Baixo-médio & Médio-alto \\
Sustentação & Atenção constante & Fácil manutenção \\
Redução de tempo & $40-70 \%$ & Mais de 100\% \\
\hline
\end{tabular}

Fonte: Adaptado de Mileham et al. (1999). 


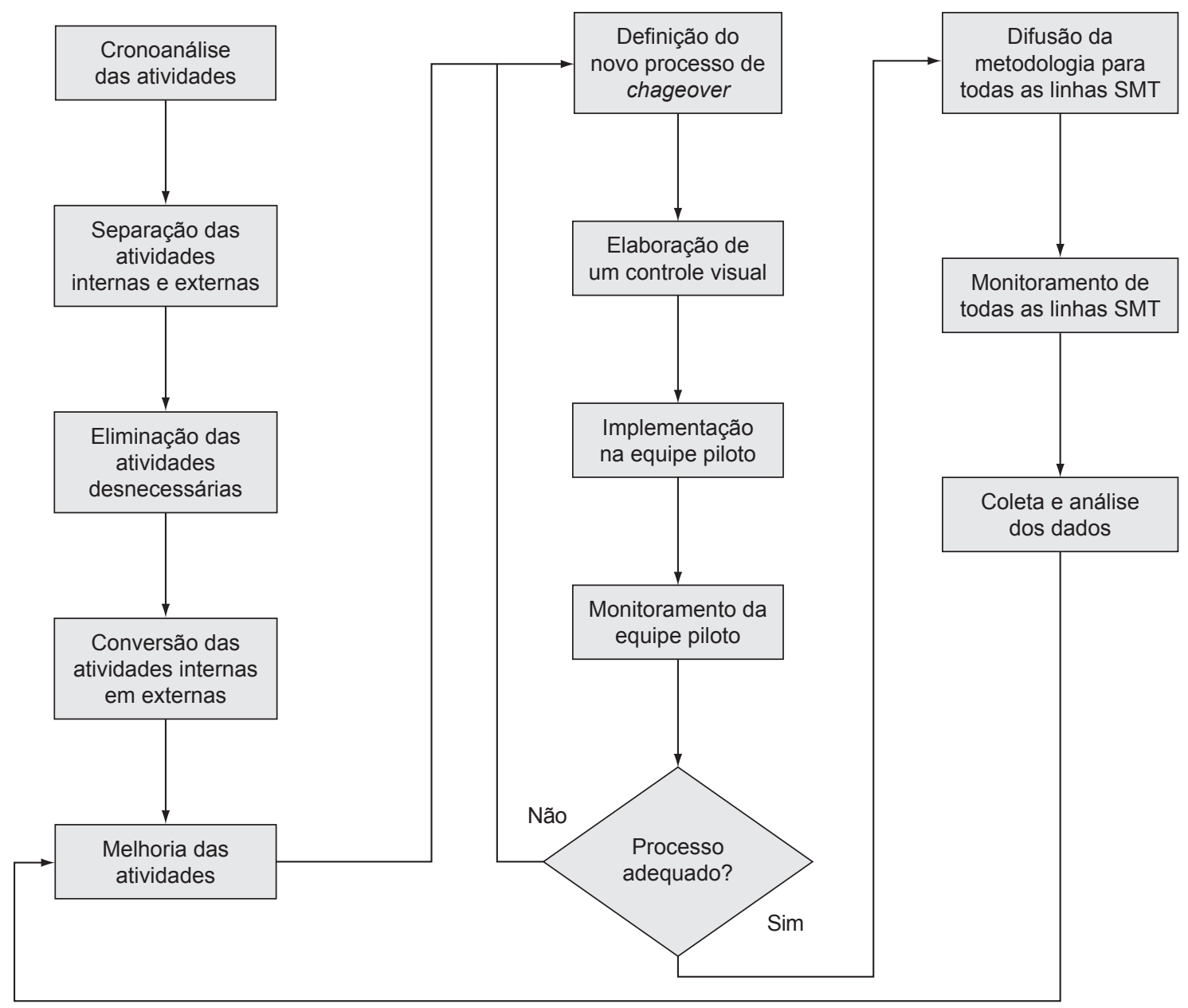

Figura 1. Metodologia.

SMED para o caso específico dos ambientes de manufatura contratada (Figura 2), referente às etapas 2, 3 e 4.

Dentro do escopo pré-determinado, o estudo de redução do changeover se fez nas linhas de produção SMT. Essa tecnologia é caracterizada pelo uso da tecnologia que insere os componentes SMD (Surface Mount Design) automaticamente na superfície das placas de circuito impresso. A escolha de atuação no SMT se deu, principalmente, por ser uma etapa da produção com alto conteúdo tecnológico, em que as máquinas são compartilhadas pela maioria dos produtos fabricados, aumentando as oportunidades de melhoria na redução do tempo de changeover. A área de produção SMT é composta por oito linhas de produção, sendo que cada uma possui basicamente cinco máquinas: uma impressora (printer), três Insersoras de componentes e um Forno. A impressora é responsável pela deposição de pasta de solda na placa onde os componentes vão ser afixados. As Insersoras se classificam em duas maneiras: insersora de componentes pequenos e em alta velocidade e insersora de componentes grandes em velocidade menor. O Forno tem a função de solidificar a pasta de solda depositada para fixar os componentes na placa. As atividades de changeover da impressora são: mudança de Stencil, reprogramação, reabastecimento de pasta de solda, ajuste de conveyor, ajuste visual de fiduciais e mudança do apoio da placa. Já as atividades de changeover das máquinas insersoras são: reprogramação, ajuste de conveyor, mudança dos alimentadores e troca dos apoios, e as do Forno são: reprogramação e ajuste de conveyor.

Um estudo preliminar foi realizado e constatou-se que o processo era executado com a ocorrência de inúmeros improvisos. Havia problemas referentes à falta de padronização das tarefas, excesso de movimentação e de ajustes.

Primeiramente, os apontamentos do tempo total despendido no changeover eram feitos por operários que, muitas vezes, sequer sabiam o seu verdadeiro conceito, fazendo com que não houvesse a forma exata de sua medição. Portanto, o apontamento dos dados para verificar o real impacto do processo na produtividade não era confiável.

A organização do trabalho para a execução das atividades era inexistente, sem nenhuma padronização ou 


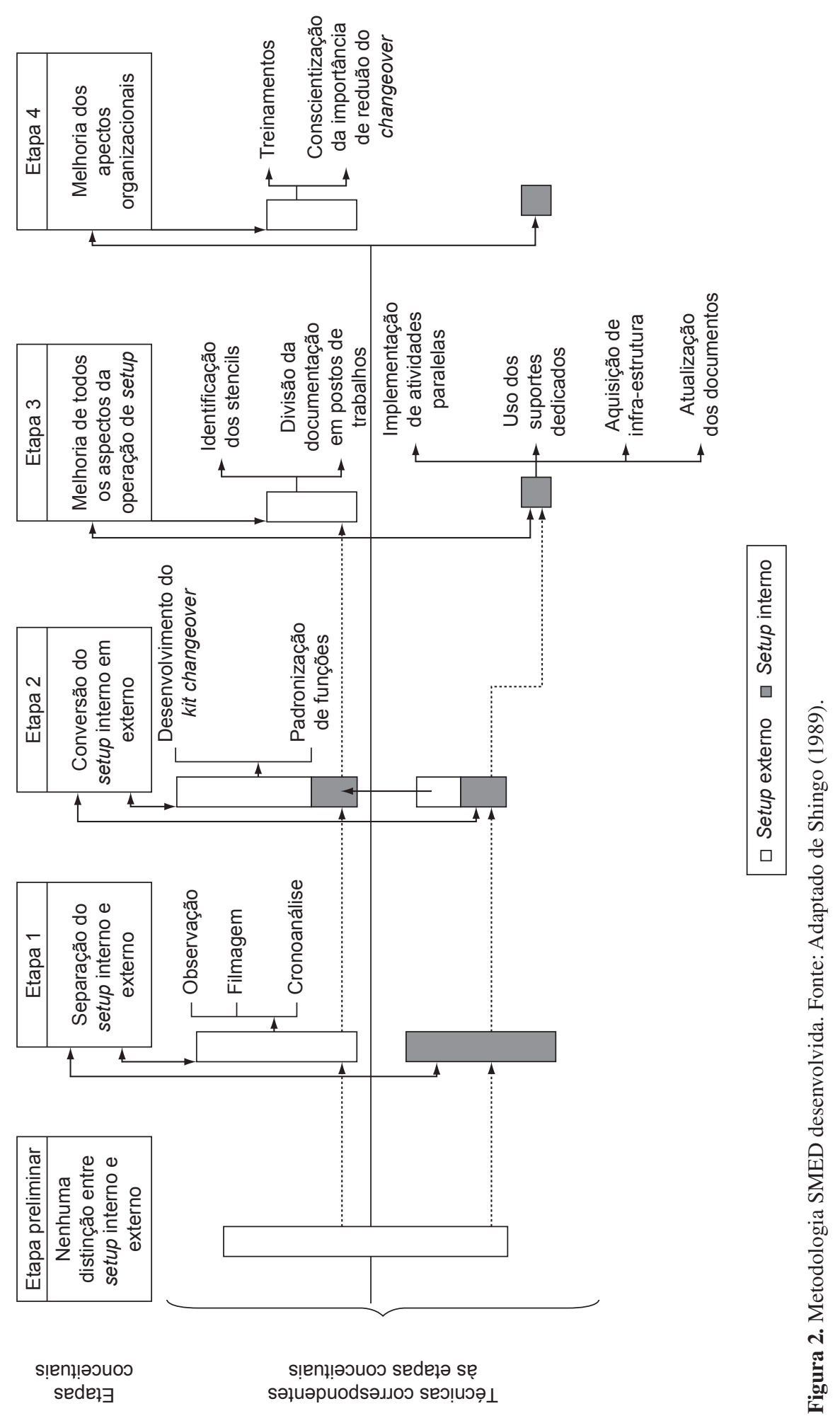


mesmo definição de responsabilidades. Esse aspecto, juntamente com a ausência de um lugar apropriado para alocação dos utensílios durante o processo, forçava movimentações intensas, afetando diretamente o tempo gasto no changeover.

Outra atividade que incrementava o tempo de parada de linha na troca de produção era uso de partes individuais na montagem de apoios da placa e ferramentas de ajustes manuais. Com essas características do processo, demonstrando sua precariedade, além da busca por competitividade num ambiente de demanda volátil e pressões dos clientes (OEM) foi constatada a necessidade de intervenção para sua melhoria. Esse objetivo foi reforçado com a análise do mapa de fluxo de valor (value stream mapping), quando foi constatado que a redução do tempo de changeover possibilitaria à empresa lidar melhor com um dos seus graves problemas: a flexibilidade de mudar rapidamente entre produtos.

Inicialmente, foi formada uma "equipe piloto" para participar da elaboração do novo processo de changeover, objetivando definir sua sequência tecnológica, assim como a padronização e rotina de trabalho.

O conhecimento do processo de changeover executado se deu por meio de observação e estudo de tempos e movimentos de suas atividades. Uma atividade complementar realizada, como sugerem Monden (1994) e Van Goubergen (2000), foi a filmagem, possibilitando análise minuciosa do processo e garantia de que todas as atividades eram executadas internamente.

Durante a etapa de conversão do setup interno em externo, as tarefas de preparação e limpeza de materiais foram todas transformadas em externas, necessitando-se de um local para alocação deles. Para isso, desenvolveu-se um suporte móvel, denominado como kit changeover, ilustrado na Figura 3. O objetivo do kit era armazenar os itens de consumo durante o changeover da impressora, possibilitando redução de movimentação do operador.

As figuras 4 e 5 detalham o dispositivo mecânico e as partes individuais utilizadas no kit changeover. $\mathrm{O}$ dispositivo e as partes mecânicas têm o objetivo de reduzir o tempo de changeover durante a troca de produtos.

Também foi desenvolvido um dispositivo de identificação dos Stencils, Figura 6, orientando os trabalhadores sobre a posição correta de inserção deles na máquina, economizando tempo e eliminando possíveis erros. Outros erros também puderam ser evitados com a atualização da documentação utilizada na fabricação dos diferentes tipos de placas.

A identificação dos Stencils acima citada também pode ser considerada como melhoria externa, pois facilitou sua localização no momento de preparação dos materiais para a troca de produção. Ainda, externamente, foi modificado o formato da documentação em que constam os procedimentos de fabricação dos produtos.
A existência de uma documentação individual por posto de trabalho evita a competição de tal documento em caso de necessidade simultânea. Economia de tempo também foi possível com a criação de atividades paralelas e organização do trabalho.

A instalação de lupa para inspeção ao lado da impressora possibilitou eliminar mais movimentos do operador, já que esta era feita longe do seu posto de trabalho.

O uso de um dispositivo sonoro foi usado para alertar os operadores sobre o início da atividade de changeover. Paralelamente, foi elaborado um tipo de controle visual, contendo, basicamente, as instruções da sequência das atividades de cada operador em cada estação de trabalho durante a execução do changeover.

\subsection{Análise dos resultados}

Os dados históricos da empresa apontam que o tempo médio de changeover era de aproximadamente 27 minutos Com a implementação do novo processo, o tempo para a realização do changeover foi reduzido para 15 minutos, em média. Esse tempo deve ser gradualmente reduzido à medida que melhorias sejam introduzidas no processo e que todos estejam adaptados à nova metodologia. Um aspecto importante constatado foi um ligeiro aumento do tempo médio de changeover logo após a implementação da metodologia SMED desenvolvida. Isto se deve ao período de adaptação dos trabalhadores às mudanças introduzidas.

O gráfico com o tempo médio de changeover da linha teste está ilustrado na Figura 7. Ele compreende uma avaliação dos dados referentes ao período de junho/05 a janeiro/06 e foram gerados pela divisão do tempo total gasto com changeover durante a semana pelo correspondente número de ocorrências.

Um fator relevante e de alto impacto no resultado do changeover é a sequência de produtos a serem fabricados. Geralmente, aos produtos que passam pelo SMT, são inseridos componentes dos dois lados, de forma não simultânea, os quais são denominados lado superior (top side) e lado inferior (back side). Esses são tratados como produtos diferentes no sequenciamento. Quando acontece de a produção dessas placas serem programadas sequencialmente, o tempo de execução do processo estudado torna-se consideravelmente reduzido. Isto porque elimina muitas etapas como ajuste de conveyor e, geralmente, troca de pinos de apoio da impressora e demais insersoras. Existem, também, produtos semelhantes que se comportam como os últimos mencionados. A semelhança desses produtos pode estar no tamanho ou na posição de inserção de componentes.

Além dos benefícios citados anteriormente, a implantação da metodologia desenvolvida também permitiu um retorno financeiro com o aumento da disponibilidade de equipamentos. Considerando-se que são realizados, em média, 80 changeovers na semana em todas as linhas SMT, e que foram economizados 12 minutos em cada 


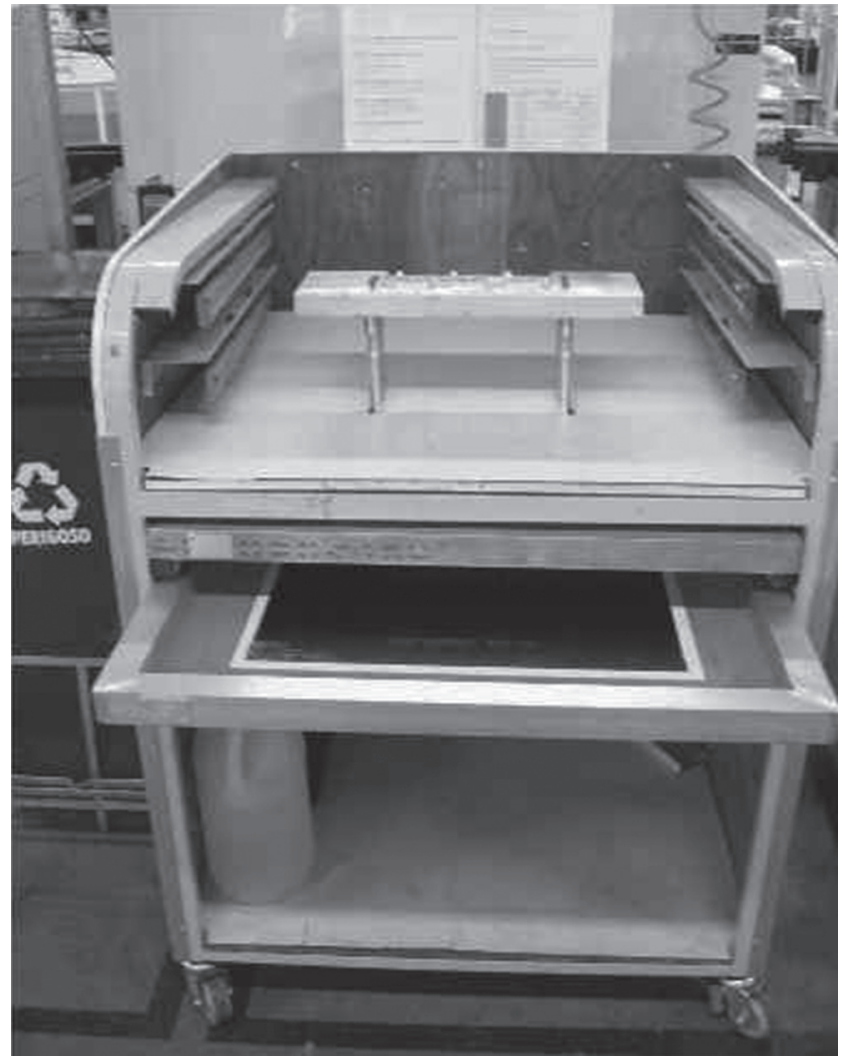

Figura 3. Kit Changeover.

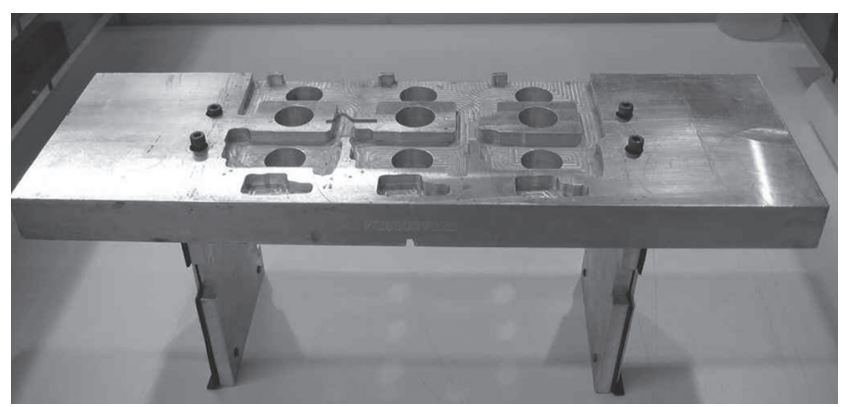

Figura 4. Dispositivo mecânico.

changeover, isso representa um aumento de 16 horas de disponibilidade dos equipamentos semanalmente. Considerando-se a hora de produção equivalente a U\$ 600,00, o faturamento semanal extra é de U\$9600,00, ou seja, R \$ 19680,00. Além dos ganhos financeiros, houve também um ganho em termo do tempo de atendimento da demanda conhecido como takt-time, isto é, houve uma melhora no nível de serviço referente ao tempo exigido pelo cliente OEM para a entrega do produto.

Os gastos com o desenvolvimento do novo processo de changeover foram de aproximadamente $\mathrm{R} \$ 25.000,00$. Portanto, contrabalançando os custos despendidos com a possibilidade de ganho, fica claro o interesse em otimizar tal processo. Fazendo uma análise mais profunda, a empresa conta também com a chance de produzir lotes menores,

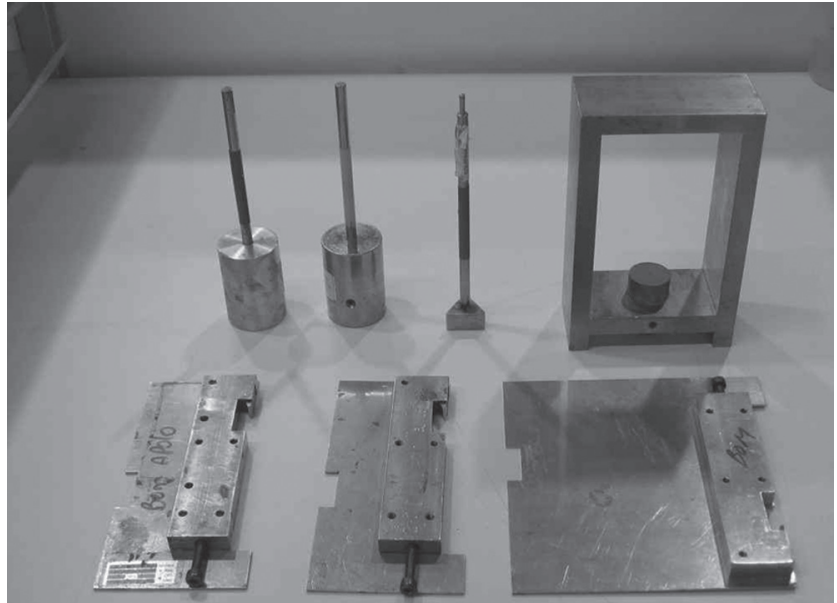

Figura 5. Partes Individuais.

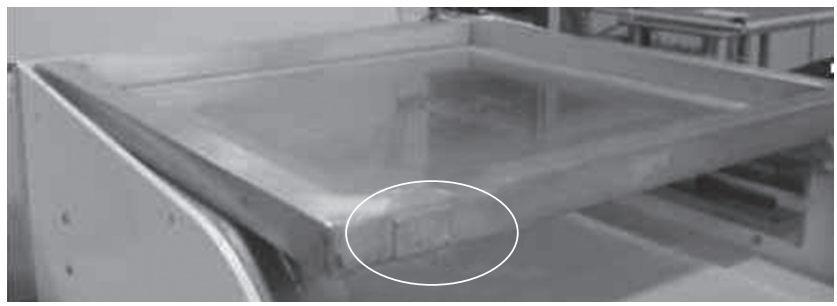

Figura 6. Identificação do Stencil.

pequenos lead times, baixo nível de estoque, assegurando melhor a qualidade dos produtos, além de aumentar a flexibilidade e, consequentemente, a competitividade frente aos concorrentes.

Deve-se ressaltar que a redução do tempo médio de changeover foi alcançada principalmente com a organização e padronização do processo, ou seja, o objetivo foi criar uma metodologia até então inexistente para a realização de tal processo.

Para se conseguir melhorias em maiores proporções, sabe-se que é necessário interferir mais profundamente na estrutura dos equipamentos, ferramentas ou produtos. Porém, essa melhoria consome muito tempo, geralmente representa altos investimentos e nem sempre a relação custo-benefício é compensadora. Quando se trata de empresas de manufatura contratada do setor eletroeletrônico, a existência de uma enorme instabilidade da demanda, caracterizada por uma alta velocidade de inovações em modelos de produtos e tecnologia empregada, elevam ainda mais os riscos de aplicação do capital para se atingir tempos menores na execução do changeover.

\section{Implicações práticas e teóricas do estudo}

Com a implementação do novo processo de changeover ficou constatada a influência da variedade dos produtos 
Tempo médio de changeover - linha 1

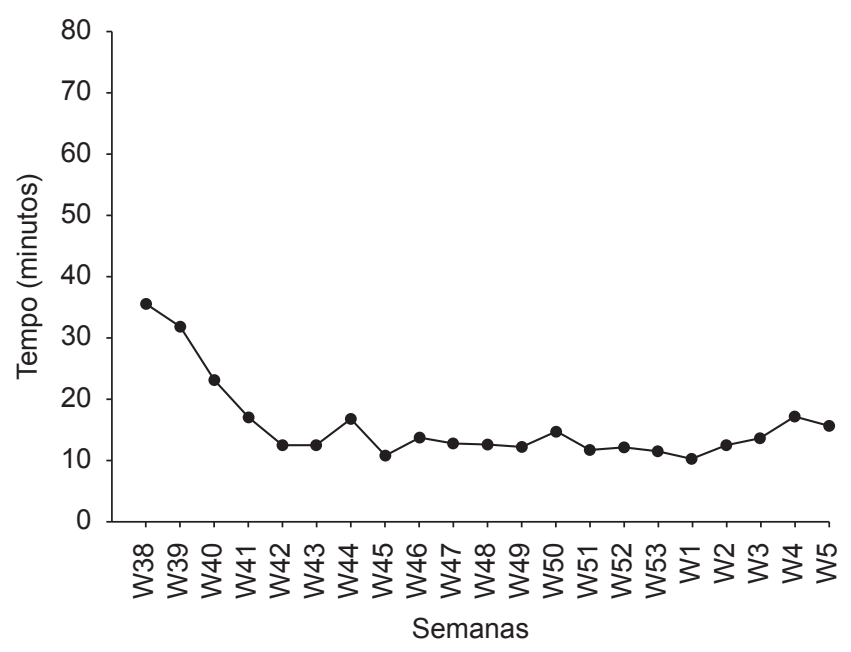

Figura 7. Tempo médio de changeover na linha 1.

produzidos e a forma como a produção era sequenciada no tempo médio gasto com essa realização. Quanto mais diferenças (tamanho, quantidade, tipo e posição dos componentes a serem inseridos) entre os produtos produzidos consecutivamente, maiores as variabilidades no processo produtivo. Isto pode ocasionar um tempo extra na estabilização da produção. Essa observação torna evidente a necessidade de se trabalhar no sequenciamento da produção, ou seja, na ordem em que os produtos serão produzidos, com o intuito de atingir o tempo médio total despendido na atividade de changeover.

As empresas do setor eletroeletrônico estão inseridas em um mercado dinâmico, levando-se em consideração o tipo de demanda, os produtos fabricados e o papel das empresas de manufatura contratada. Os clientes estão interessados em produtos diferenciados, de alta qualidade, acessíveis financeiramente e com curto prazo de entrega.

Atualmente, os produtos são caracterizados por serem "mundiais", ou seja, fabricados localmente e comercializados mundialmente, o que agrava a concorrência, principalmente com países como o México, Índia ou China. Desenvolver sistemas de produção enxutos com fortes características de flexibilidade é uma condição necessária para evitar a migração das plantas industriais para países emergentes que atualmente competem fortemente com o Brasil pela localização das EMSs. Em se tratando dos produtos eletrônicos, esses, ainda, são caracterizados por um alto valor agregado e por um curto ciclo de vida, sofrendo modificações no produto e no processo, inovações e rupturas tecnológicas numa velocidade surpreendente. Esses aspectos tendem a forçar à produção de uma grande variedade de produtos, porém em pequenas quantidades. Além das características relatadas anteriormente, definindo o cenário das empresas do setor eletroeletrônico, aquelas do tipo de manufatura contratada ainda sofrem um agravante que é a constante pressão por parte das OEMs pela redução de custos, aumento de produtividade e qualidade dos serviços e produtos. É muito comum atender diversas OEMs, aumentando ainda mais a necessidade de fabricação de um enorme mix de produtos.

Portanto, esse dinamismo significa que as empresas, para garantir a competitividade, devem ser flexíveis e capazes de responder rapidamente às demandas do mercado.

A manufatura enxuta surge então como uma possibilidade de assegurar essa agilidade ao proporcionar um processo produtivo mais eficiente e estável, já que predomina a eliminação de quaisquer atividades que não agregam valor ao produto final, melhorando o nível de serviço aos clientes e, consequentemente, à concorrência.

Dentro dessa filosofia de produção, existem várias técnicas para melhoria da organização como um todo. Porém, o processo de transformação e adaptação a essa modalidade requer empenho, determinação, estabelecimento claro dos objetivos e motivação de todos, principalmente da alta gerência para conduzir tal evento.

Um dos pré-requisitos para se atingir a flexibilidade do sistema produtivo, inclusive das empresas produtoras de PCBs (Printed Circuit Boards - placas de circuito impresso), segundo Cyr et al. (1997), é a redução do tamanho dos lotes de produção, que está condicionada à redução do tempo médio do setup/changeover realizado. A aplicação do SMED, resultando na diminuição do tempo de parada das linhas de montagem quando houver troca de produção, possibilita também um lead time menor, níveis de estoques mais baixos e aumento da disponibilidade das máquinas e, consequentemente, aumento da produtividade. Por sua vez, um lead time reduzido com a fabricação de pequenas quantidades, garante à empresa maiores chances de entregar os produtos no momento exato. Adicionalmente, favorece o processo de inserção de um novo produto no mercado, com capacidade de disponibilizá-lo mais rapidamente. Esses fatores possibilitam maior poder de resposta da empresa às variações da demanda, que são comuns ao setor do qual faz parte. E ainda, níveis de estoques baixos permitem visualizar problemas de qualidade mais prontamente, reduzindo custos com retrabalho e obsolescência tecnológica de produtos. Outro aspecto importante é que o setup/changeover, quando realizado corretamente, previne não conformidades no processo, assegurando a confiabilidade do processo e qualidade aos produtos. A confiabilidade pode ser mensurada pela qualidade dos produtos gerados ou pelo cumprimento aos prazos de entrega estabelecidos (CYR et al., 1997).

Uma outra característica presente das empresas de manufatura contratada é a existência de clientes que correspondem a um percentual significativo das vendas. Isto ocasiona um risco considerável à empresa, em caso de desistência do contrato. Portanto, a habilidade de lidar com alterações de variedade e de volume, incorre em maiores possibilidades de fidelização do cliente, concedendo uma 
certa estabilidade à empresa prestadora de serviço de manufatura.

Com isso, a implantação de metodologias de redução do tempo de changeover torna-se essencial à sobrevivência das empresas de manufatura contratada, já que lhes permite se adequarem ao tipo de demanda volátil dos produtos que fabricam e às exigências dos clientes, OEMs e consumidores finais.

\section{Conclusões}

Mercados dinâmicos, como o do setor eletroeletrônico, caracterizado pela fabricação de produtos de alto valor agregado com curto ciclo de vida, demanda instável, concorrência internacional, forte presença de empresas do tipo de manufatura contratada e fortes perspectivas de crescimento, têm exigido das empresas flexibilidade do processo produtivo, baixos custos e capacidade de resposta rápida às variações da demanda.

A redução de changeover é o pré-requisito para possibilitar a produção em pequenos lotes. É notável que essa diminuição da quantidade produzida aumente o número de ocorrências de tal processo. Portanto, a metodologia implementada também tem a função de equilibrar essa situação à medida que permite uma troca de produção mais ágil.

Outra característica importante é a tendência dessas empresas na produção de grandes variedades de produtos em pequenos volumes de produção, denominadas de HMLV(High-mix, Low-volume).

As empresas de manufatura contratada (EMS), responsáveis pela fabricação de produtos das empresas detentoras da marca (OEMs), sofrem pressões para aumento de produtividade, redução de custos, respostas rápidas às variações da demanda e curto ciclo de produção, permitindo a introdução rápida de produtos no mercado. Portanto, aquela que oferecer um sistema produtivo enxuto dotado de fortes características de flexibilidade terá maiores chances de se manter no mercado, onde a concorrência é mundial, acirrada e crescente.

Nesse sentido, a produção enxuta, então, surge como uma alternativa no alcance de vantagens competitivas por permitir uma produção de grande variedade de produtos, caracterizada por um curto lead time, alto nível de qualidade e eficiente uso dos recursos.

A implementação da técnica SMED possibilitou reduções significativas do tempo médio despendido. Esses resultados foram conseguidos por meio da organização e padronização do processo, com pouca utilização de investimentos e retorno significativo para a empresa.

O desenvolvimento e a implantação do SMED evidenciaram que a participação de todos da empresa é essencial para a consolidação da política de produção enxuta. O comprometimento da alta gerência é fundamental na busca constante de motivação da equipe.

De forma geral, pela análise das implicações teóricas e práticas apresentadas na seção anterior, a adoção da técnica SMED, com um curto changeover, possibilita um processo produtivo mais estável, aumento de produtividade e redução de custos, elementos fundamentais para uma empresa de manufatura contratada ser competitiva em ambientes de produção complexos e dinâmicos. Além disso, permite a produção de um grande mix de produtos, com a redução dos tamanhos dos lotes de produção e maior agilidade para responder às variações da demanda, com a redução dos lead times.

\title{
Development and implementation of a SMED methodology in contract manufacturing enviroments
}

\begin{abstract}
This paper presents the development of a methodology to reduce the machine preparation time (changeover time) in volatile environments of the informatics and electronic contract manufacturing. The companies of this sector produce a large product variety characterized for a high aggregate value, short life cycle, and demand instability requiring flexibility to follow the dynamism of the market. Thus, the lean manufacturing seems to be a good alternative since its main characteristics are: continuous production flow, high quality level, low costs, and fast changes' introduction. The focus of this study is to reduce tool change time in a production line that uses the SMT (Surface Mount Technology). Time reduction is extremely important because it leads to the reduction of intermediate inventor, increases production flexibility with short lots and lead times, productivity increase, fast responses to improvements of the amount and production programming. The methodology used was the Action Research. The paper brings theoretical and practical contributions besides the application of the developed methodology in the contract manufacturing sector. The method developed allowed flexibility to respond to variety and volume changes resulting in considerable financial, productivity, and flexibility gains.
\end{abstract}

Keywords: Changeover reduction. Lean manufacturing. Contract manufacturing. 
AGUSTIN, R.; SANTIAGO F. Single-Minute Exchange of Die. In: IEEE/SEMI ADVANCED SEMICONDUCTOR MANUFACTURING CONFERENCE, 1996. Proceedings... Piscataway, NJ: IEEE Conference, 2006. p. 214-217.

ÅHLSTRÖM, P. Sequences in the implementation of lean manufacturing. European Management Journal, v. 16, n. 3, p. 327-334, 1998.

BAYOU, M. E.; KORVIN, A. Measuring the leanness of manufacturing system: A case study of Ford Motor Company and General Motors. Journal of Engineering and Technology Management, v. 25, n. 4, p. 287-304, 2008.

BOYLE, T. A.; SCHERRER-RATHJE, M. An Empirical Examination of the Best Practices to Ensure Manufacturing Flexibility - Lean Alignment. Journal of Manufacturing Technology Management, v. 20, n. 3, p. 348-366, 2009.

CHAN, M. F. S.; CHUNG, W. C. W. A framework to develop an enterprise information portal for contract manufacturing. International Journal of Production Economics, v. 75, p. 113-126, 2002.

CHECKLAND, P. Systems Thinking, Systems Practice. New York: John Wiley e Sons, 1981.

CHECKLAND, P.; SCHOLES, J. Soft Systems Methodology in Action. Chichester: John Wiley e Sons, 1990.

COUGHLAN, P.; COGHLAN, D. Action research for operations Management. International Journal of Operations e Production Management, v. 22, n. 2, p. 220-240, 2002.

COUPRIE, D. et al. Soft Systems Methodology. Calgary: Departament of Computer Science, University of Calgary, 1997. Disponível em: http://www.cpsc.ucalgary.ca/cpsc_research/areas/evolutionary. Acesso em: 10 Junho 2009.

CYR, B. et al. Manufacturing flexibility: SMT factors study. Computers and Industrial Engineering, v. 33, n. 1-2, p. 361-364, 1997.

DIABY, M. Integrated batch size and setup reduction decisions in multiproduct, dynamic manufacturing environments. International Journal of Production Economics, v. 67, n. 3, p. 219-233, 2000.

FARLOW, D. Efficient Line Changeover. SMT Magazine, v. 19, n. 3, p. 44-45, março 2005.

HADAYA, P.; LEFEBVRE, E.; LÉGER, P. Roles and strategies of contract manufacturers in the telecommunications industry. In: IEEE, 2000. Proceedings... Piscataway, NJ: IEEE, 2000. p. $458-463$.

HALL, R. W. Zero inventories crusade-much more than materials management. Production and Inventory Management Journal, v. 24, n. 3, p. 1-8, 1983.

HEALE, G. Applying theory to practice: an action research resource pack for professionals. Clinical Chiropractic, v. 6, n. 1, p. 4-14, 2003.

HERRON, C.; HICKS, C. The transfer of selected lean manufacturing techniques from Japanese automotive manufacturing into general manufacturing (UK) through change agents. Robotics and ComputerIntegrated Manufacturing, v. 24, n. 4, p. 524-531, 2008.

HOLWEG, M. The genealogy of lean production. Journal of Operations Management, v. 25, n. 2, p. 420-437, 2007.

KRAFCIK, J. F. Triumph of the Lean Production System. Sloan Management Review, v. 30, n. 1, p. 41-52, 1988.

MCINTOSH, R. et al. An assessment of the role of design in the improvement of changeover performance. International Journal of Operations e Production Management, v. 16, n. 9, p. 5-22, 1996.
MILEHAM, A. R. et al. Rapid changeover - a pre-requisite for responsive manufacture. International Journal of Operations \& Production Management, v. 19, n. 8, p. 785-796, 1999.

MONDEN, Y. Toyota Production System: An integrated approach to just-in-time. London: Chapman e Hall, 1994.

MOXHAM, C.; GREATBANKS, R. Prerequisites for the implementation of the SMED methodology. International Journal of Quality e Reliability Management, v. 18, n. 4, p. 404-414, 2001. Manufacturing Market Insider - MMI. 2009. Disponível em: <http://mfgmkt.com>.

OHNO, T. O Sistema Toyota de Produção: além da produção em larga escala. Porto Alegre: Bookman, 1997.

PANIZZOLO, R. Applying the lessons learned from 27 lean manufacturers. The relevance of relationships management. International Journal of Production Economics, v. 55, n. 3, p. 223-240, 1998.

PHAM, D. T.; PHAM, P. T. N.; THOMAS, A. Integrated production machines and systems - beyond lean manufacturing. Journal of Manufacturing Technology Management, v. 19, n. 6, p. 695-711, 2008.

SALNER, M. Beyond Checkland e Scholes: Improving SSM. In: INTERNATIONAL CONFERENCE OF THE SYSTEM DYNAMICS SOCIETY AND AUSTRALIAN e NEW ZEALAND SYSTEM CONFERENCE, 17, 5, 1999, New Zealand, Wellington, Anzsys. Proceedings...

SCHONBERGER, R. J. Japanese Manufacturing Techniques. New York: The Free Press, 1982.

SEVERSON, D. The SMED system for reducing changeover times: an exciting catalyst for companywide improvement and profits. P\&IM Review with APICS News, v. 8, n. 10, p. 10, 15-16, 1988.

SHAH, R.; WARD, P. T. Defining and developing measures of lean production. Journal of Operations Management, v. 25, n. 4, p. 785-805, 2007.

SHARMA, V. SMED for high-mix assembly. Circuits Assembly, v. 12 , n. 1, p. 62, 64-68, 2001.

SHINGO, S. A Study of the Toyota Production System - from an industrial engineering viewpoint. Portland: Productivity Press, 1989.

SHINGO, S. Non-stock production: the Shingo system for continuous improvement. Cambridge: Productivity Press, 1988.

STURGEON, T. J. Turnkey production networks: a new american model of industrial organization? Berkeley: Berkeley Roundtable on the International Economy, University of California, 1997.

TANEL, G. Acquisition trends in EMS. Surface Mount Technology, v. 19, n. 7 , p. 32, julho 2005.

UPTON, D. M. Flexibility as process mobility: the management of plant capabilities for quick response manufacturing. Journal of Operations Management, v. 12, n. 3-4, p. 205-224, 1995.

Van GOUBERGEN, D. Set-up reduction as an organization-wide problem. In: SOLUTIONS 2000 CONFERENCE, 2000. Proceedings... Cleveland: DRI Research e Publications, 2000.

Van GOUBERGEN, D.; Van LANDEGHEM, H. Rules for integrating fast changeover capabilities into new equipment design. Robotics and Computer Integrated Manufacturing, v. 18, n. 3-4, p. 205-214, 2002.

WEBER, A. Contract Manufacturing On the Rise. Assembly Magazine, setembro 2002. Disponível em: <http://www.cmcseattle.com/ newsfolder/assembly-02.htm>. Acesso em: 24 Fevereiro 2006.

WOMACK, J. P.; JONES, D. T.; DANIEL, R. A máquina que mudou o mundo. Rio de Janeiro: Editora Campus, 1992. 


\section{Samuel Vieira Conceição}

Departamento de Engenharia de Produção, Universidade Federal de Minas Gerais - UFMG Avenida Antonio Carlos, 6627 - Campus Pampulha, CEP 31270-901, Belo Horizonte - MG e-mail: svieira@dep.ufmg.br

\section{lana Araújo Rodrigues}

Departamento de Engenharia de Produção, Universidade Federal de Minas Gerais - UFMG Avenida Antonio Carlos, 6627 - Campus Pampulha, CEP 31270-901, Belo Horizonte - MG e-mail: iana.araujo@gmail.com

\section{Andressa Amaral Azevedo}

Departamento de Engenharia Mecânica, Universidade Federal de Minas Gerais - UFMG Avenida Antonio Carlos, 6627 - Campus Pampulha, CEP 31270-901, Belo Horizonte - MG e-mail: dressa.azevedo@gmail.com

\section{João Flávio Almeida}

Departamento de Engenharia de Produção, Universidade Federal de Minas Gerais - UFMG Avenida Antonio Carlos, 6627 - Campus Pampulha, CEP 31270-901, Belo Horizonte - MG e-mail: joaoflavio.ufmg@gmail.com

\section{Adriano Morais}

Jabil Indústria Eletro-Eletrônico do Brasil e-mail: adriano_morais@jabil.com

\section{Fabrício Ferreira}

Jabil Indústria Eletro-Eletrônico do Brasil e-mail: fabricio_ferreira@jabil.com 